\title{
Diabetes Insipidus Induced by Combination of Short-acting Octreotide and Lanreotide for Recurrent Carcinoid Crisis of Neuroendocrine Tumour: A Case Report
}

\author{
Goh Kian Guan, ${ }^{1}$ Subashini Rajoo, ${ }^{1}$ Noraini Mohd Dusa, ${ }^{2}$ \\ Nik Hasimah Nik Yahya, ${ }^{2}$ Mohamed Badrulnizam Long Bidin ${ }^{1}$ \\ ${ }^{1}$ Department of Medicine, Hospital Kuala Lumpur, Wilayah Persekutuan Kuala Lumpur, Malaysia \\ ${ }^{2}$ Histopathology Unit, Department of Pathology, Hospital Kuala Lumpur, Malaysia
}

\begin{abstract}
Somatostatin analogue is useful in carcinoid crisis for symptom control. Optimal dosing of somatostatin analogues for carcinoid symptoms is not known. This case highlighted management issues using combination short-acting octreotide infusion with long-acting lanreotide during carcinoid crisis. The patient had left lung neuroendocrine tumour that metastasized to his liver and bone, post left lobectomy. Due to extensive metastasis to the liver causing recurrent carcinoid crisis, he required shorter interval long-acting lanreotide with continuous infusion of short-acting octreotide, which led to transient diabetes insipidus. Symptoms resolved with discontinuation of treatment. Somatostatin analogues, especially in combination, may inhibit the posterior pituitary resulting in diabetes insipidus. Prompt withdrawal of shortacting somatostatin analogue and initiation of desmopressin can reverse the complication. It is important to recognize this complication with combination of octreotide and lanreotide injections to avoid serious complications.
\end{abstract}

Key words: diabetes insipidus, octreotide, lanreotide, neuroendocrine tumors, malignant carcinoid syndrome

\section{INTRODUCTION}

Neuroendocrine tumour (NET) is rare malignancy with the potential to secrete bioactive amines resulting in carcinoid syndrome. Pulmonary NET occurred in 0.2 to 2/100,000 population/year in Western countries. ${ }^{1}$ Carcinoid syndrome occurred in $19 \%$ of NET, which was associated with shorter overall survival. ${ }^{2}$ In carcinoid crisis, there is over-secretion of biochemically-active peptides leading to flushing, hypotension, severe hypertension, diarrhoea and acidosis. Somatostatin analogue is used to control carcinoid symptoms in NET. Despite this, some patients still experience breakthrough of carcinoid symptoms, thus requiring a higher dose or alternative strategies. However, there is no optimal dosing recommended so far to overcome carcinoid crisis. Therefore, dose related adverse effects are not frequently sighted or reported in the past. This case highlighted the rare complication of diabetes insipidus in metastatic lung neuroendocrine tumour treated with combination of high-dose somatostatin analogues and understanding the pathophysiology behind it. It is important to recognize this complication, as it is reversible and treatable.

\section{CASE}

We present a case of a 62-year-old male who presented with carcinoid syndrome such as recurrent vomiting, diarrhoea, facial flushing, palpitation and unintentional weight loss for two months. Investigation to look for the source of carcinoid syndrome included CT scan of thorax, abdomen and pelvis which showed a heterogeneouslyenhancing lobulated mass at the laterobasal segment of left lower lobe of lung (largest diameter $9.5 \mathrm{~cm}$ ) with multiple large liver lesions (largest diameter $8.2 \mathrm{~cm}$ ) and bone involvements. Urine homovanillic acid pre-operatively was elevated to $149.94 \mathrm{mg}$ per $24 \mathrm{~h}$ (0-34.3 mg per $24 \mathrm{~h})$. Urine 5-hydroxyindoleacetic acid was similarly raised to $3677.9 \mathrm{mg}$ per $24 \mathrm{~h}$ (3.6-42.89 mg per 24h). Biopsy of lung was negative for malignancy cells. Baseline Galium-68 DOTANOC scan showed focal uptakes at the area of the lung mass, at left lower lobe and heterogenous uptake in both liver lobes and vertebral body. Ultrasound-guided liver biopsy showed well-differentiated NET.

He underwent left lower lobe lobectomy and histopathology confirmed to be NET with synaptophysin positivity and $\mathrm{Ki}-67<2 \%$ (Grade 1) (Figures 1 and 2). Intramuscular lanreotide $120 \mathrm{mg}$ was initiated 1-month post lobectomy and continued 4-weekly. Four months post somatostatin analogue therapy, Ga-68 DOTANOC PET CT showed persistent somatostatin-avid lesions in liver and bone. Chromogranin A level was elevated at $1960.78 \mathrm{nmol} / \mathrm{L}$ (0.79-2.74 nmol/L) and reduced marginally to 1414.72 $\mathrm{nmol} / \mathrm{L}$ after surgery. His symptoms persisted requiring multiple admissions, hence, lanreotide injection frequency was shortened to bi-weekly. Despite that, there were multiple carcinoid crises breakthrough requiring monthly admissions. Each time, he received continuous, escalating octreotide infusion during admission of carcinoid crisis 
to control his symptoms of palpitations, hypotension and flushing. Subsequently, his urine output increased to $300 \mathrm{cc}$ per hour $(4.6 \mathrm{ml} / \mathrm{kg} /$ hour) (serum osmolarity: 295 mOsm, urine osmolarity: 213 mOsm and serum sodium $146 \mathrm{mmol} / \mathrm{L}$ ) once octreotide infusion reached $75 \mathrm{mcg}$ per hour. Carcinoid symptoms finally resolved with $150 \mathrm{mcg}$ per hour of octreotide infusion. All other causes of polyuria such as hypercalcaemia, hyperglycaemia and primary polydipsia were excluded. Serum calcium was $2.25 \mathrm{mmol} / \mathrm{L}$ ( $9 \mathrm{mg} / \mathrm{dL})$. He was treated as central diabetes insipidus and was given subcutaneous desmopressin 2 mcg injections, reducing urine output to $0.5 \mathrm{ml} / \mathrm{kg} /$ hour. After resolution of carcinoid crisis, octreotide infusion was slowly tapered off and no recurrence of polyuria was observed. These carcinoid crisis episodes were recurrent, and he had a total of 4 admissions for symptoms control. Short-acting octreotide infusion had resulted in carcinoid crisis control but caused recurrent episodes of diabetes insipidus. Repeated Galium scan showed disease progression and extensive metastases (Figure 3). Peptide receptor radionuclide therapy (PRRT) was considered for the patient for disease-control and symptom-control.

\section{DISCUSSION}

The posterior pituitary is a neural tissue consisting of distal axons of hypothalamic magnocellular neurons. ${ }^{3}$ Control of posterior pituitary hormone synthesis is via messenger RNA (mRNA) transcription in the magnocellular neurons. ${ }^{4}$ The major stimulatory pathway of posterior pituitary is through the direct effect of norepinephrine on presynaptic glutamate neurons. ${ }^{5}$ The main inhibitory input is via presynaptic gamma aminobutyric acid (GABA) receptors, which can result in decreased secretion of arginine vasopressin (AVP). ${ }^{6}$ Vasopressin is the main hormone regulating osmolality and water homeostasis. Diabetes insipidus (DI) is defined as excretion of abnormally large amount of diluted urine (urine osmolality less than 300 $\mathrm{mOsm} / \mathrm{kg}$ of water). The fundamental aetiology of DI is as follows: decreased AVP secretion (cranial DI, hypothalamic disorder); decreased AVP effects (nephrogenic DI); excessive water intake (dipsogenic or psychogenic polydipsia); and increased AVP metabolism (gestational DI). ${ }^{7}$

Somatostatin is synthesized in parvicellular neurons and found to have overlapped distribution with AVP and somatostatin-like immunoreactive fibres in pituitary and neurohypophysis. ${ }^{8}$ This was supported by a study whereby somatostatin administered in increasing doses was able to reduce AVP secretion in haemorrhage-induced sheep. ${ }^{9}$ In the distal nephron, somatostatin also inhibits AVP action and increase basal water permeability, leading to diuresis. ${ }^{10}$ Octreotide and lanreotide are somatostatin receptor analogues that have multiple inhibitory effects.

The mainstay of treatment in NET is surgical resection of the tumour. Metastatic NET usually requires multimodal approaches such as liver-targeted therapy, cytotoxic chemotherapy, PRRT or medical therapy. Targeted agents such as everolimus and sunitinib had been used to improve progression-free survival. ${ }^{11}$ Somatostatin receptor ligands alleviates carcinoid symptoms by blocking multiple hormone release. ${ }^{12}$ Both octreotide and lanreotide are equally effective in controlling carcinoid symptoms. ${ }^{13}$ However, the patient showed only partial response to high

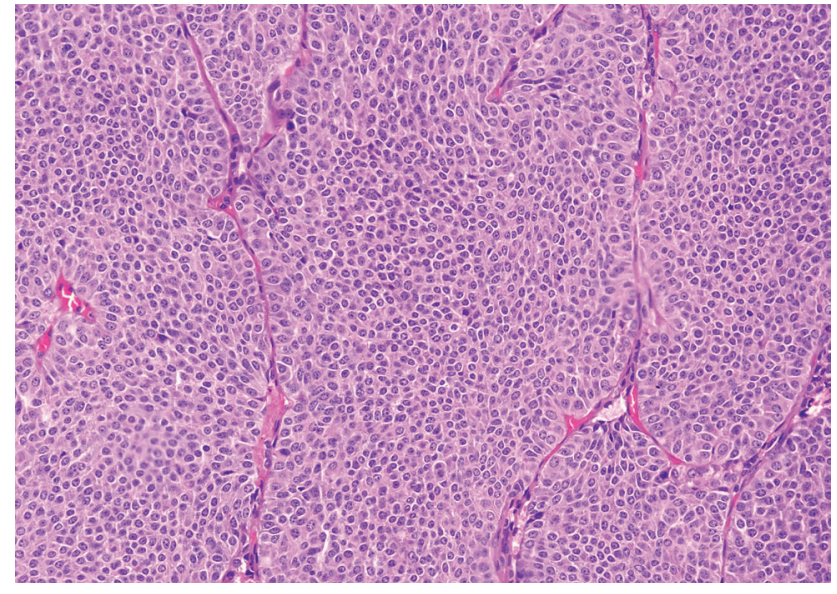

Figure 1. Tumour with uniform population of neoplastic cells with fine granular chromatin pattern and inconspicuous small nucleoli (H\&E, 200x).

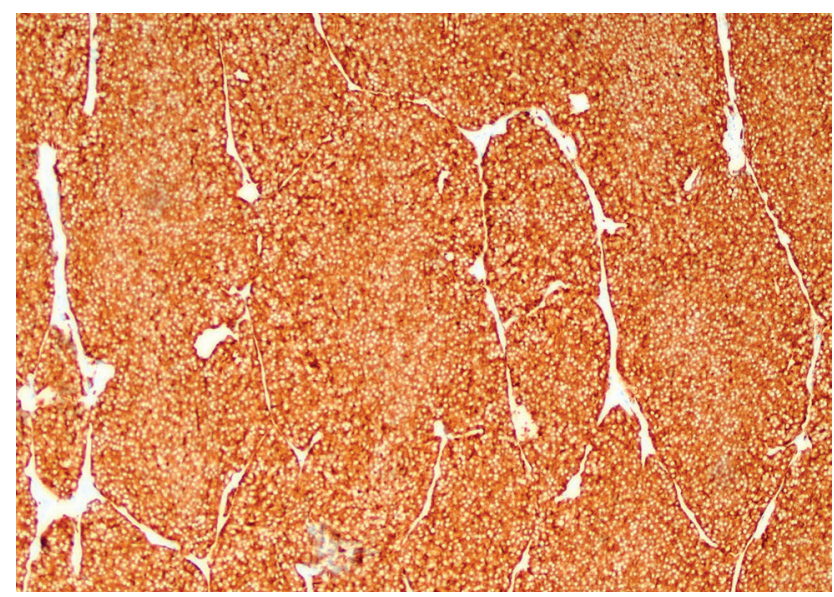

Figure 2. Tumour cells showing diffuse positivity for synaptophysin (SYN, 100x).

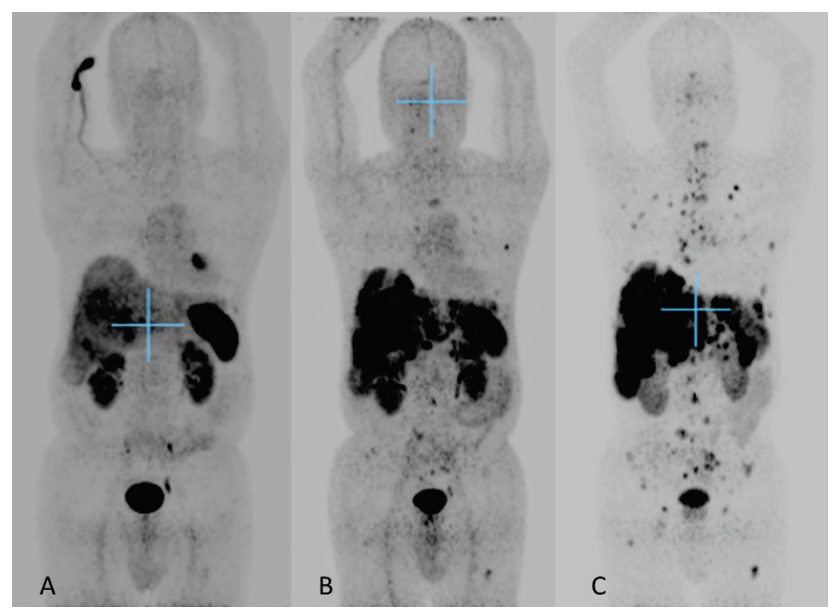

Figure 3. (A) Galium-68 DOTANOC scan showing somatostatin-avid disease in left lower lobe of lung mass and heterogenous uptake in both lobes of liver and vertebra spine; (B) Galium-68 DOTANOC imaging post left lung lobectomy and lanreotide therapy showing disease progression with increasing somatostatin-avid lesions in liver, vertebrae, pelvic, ribs and scapula; (C) Post PRRT scan showing high tumour burden with increased somatostatin-avid lesions in neck, both lung fields and abdomen. 
dose subcutaneous lanreotide (120 mg every 14 days) and required intermittent octreotide infusion (up to $150 \mathrm{mcg}$ per hour) to cover for breakthrough of acute carcinoid crisis. This approach reduced the patient's symptom severity and shortened the length of hospital stay.

In phase I trial of octreotide for NET, carcinoid symptoms were better controlled with a higher dose of octreotide and no dose-limiting adverse effects were observed with doses up to 2000 mcg q $8 \mathrm{~h}$ (total 6000 mcg per day). ${ }^{14}$ Therefore, we propose that escalating doses of octreotide infusion (up to $3600 \mathrm{mcg}$ per day) with concurrent high dose longacting lanreotide had possibly inhibited AVP release from the pituitary and disrupted AVP action at distal nephrons leading to DI.

From our literature review, this is the first case to report an association of DI with the combination of octreotide and lanreotide. The resolution of DI once octreotide infusion was tapered and stopped also suggests transient effects of somatostatins analogues on the posterior pituitary. Concurrent use of two somatostatin receptor ligands can effectively alleviate carcinoid syndrome. However, the potential complication of DI needs to be monitored and promptly treated.

\section{CONCLUSION}

Short-acting somatostatin analogue (octreotide) can be augmented with long-acting somatostatin analogue during breakthrough carcinoid crisis. However, physicians need to practice caution as the combination of somatostatin receptor analogues at high doses can result in iatrogenic DI, though this rare encounter is usually transient and reversible.

\section{Ethical Consideration}

Patient consent was obtained before submission of the manuscript.

\section{Statement of Authorship}

All authors certified fulfilment of ICMJE authorship criteria.

\section{Author Disclosure}

The authors declared no conflict of interest.

Funding Source

None.

\section{References}

1. Caplin ME, Baudin E, Ferolla P, et al. Pulmonary neuroendocrine (carcinoid) tumors: European Neuroendocrine Tumor Society expert consensus and recommendations for best practice for typical and atypical pulmonary carcinoids. Ann Oncol. 2015;26(8):1604-20. PMID: 25646366. https://doi.org/10.1093/annonc/mdv041.

2. Halperin DM, Shen C, Dasari A, et al. Frequency of carcinoid syndrome at neuroendocrine tumour diagnosis: A population-based study. Lancet Oncol. 2017;18(4):525-34. PMID: 28238592. PMCID: PMC6066284. https://doi.org/10.1016/S1470-2045(17)30110-9.

3. Oliet SHR, Piet R, Poulain DA, et al. Glial modulation of synaptic transmission: Insights from the supraoptic nucleus of the hypothalamus. Glia. 2004;47(3):258-67. PMID: 15252815. https://doi. org/10.1002/glia.20032.

4. Sherman TG, McKelvy JF, Watson SJ. Vasopressin mRNA regulation in individual hypothalamic nuclei: A Northern and in situ hybridization analysis. J Neurosci. 1986;6(6):1685-94. PMID: 3712004. PMCID: PMC6568715. https://doi.org/10.1523/JNEUROSCI.06-06-01685.1986.

5. Daftary SS, Boudaba C, Szabó K, et al. Noradrenergic excitation of magnocellular neurons in the rat hypothalamic paraventricular nucleus via intranuclear glutamatergic circuits. J Neurosci. 1998;18(24):10619-28. PMID: 9852597. PMCID: PMC6793374. https:// doi.org/10.1523/JNEUROSCI.18-24-10619.1998.

6. Kabashima N, Shibuya I, Ibrahim N, et al. Inhibition of spontaneous EPSCs and IPSCs by presynaptic GABA(B) receptors on rat supraoptic magnocellular neurons. J Physiol. 1997;504(Pt 1):113-26. PMID: 9350623. PMCID: PMC1159941. https://doi.org/10.1111/j.1469-7793. 1997.113bf.x.

7. Robertson GL. Diabetes insipidus. Endocrinol Metab Clin North Am. 1995;24(3): 549-72. PMID: 8575409.

8. Renaud LP, Bourquet CW. Neurophysiology and neuropharmacology of hypothalamic magnocellular neurons secreting vasopressin and oxytocin. Progress in Neurobiology. 1991;36(2):131-69. PMID: 1998074. https://doi.org/10.1016/0301-0082(91)90020-2.

9. Wang X, Tresham JJ, Congiu M, et al. Somatostatin centrally inhibits vasopressin secretion during haemorrhage. Brain Res. 1987;436(1):199203. PMID: 2891414. https://doi.org/10.1016/0006-8993(87)91577-0.

10. Ray C, Carney S, Morgan T, Gillies A. Somatostatin as a modulator of distal nephron water permeability. Clin Sci. 1993;84(4):455-60. PMID: 8097684. https://doi.org/10.1042/cs0840455.

11. Ishida $\mathrm{H}$, Lam AKY, pancreatic neuroendocrine neoplasms: The latest surgical and medical treatment strategies based on the current World Health Organization classification. Crit Rev Oncol Hematol. 2019;145. PMID: 31864179. https://doi.org/10.1016/j.critrevonc.2019.102835.

12. Janson ET, Oberg K. Long-term management of the carcinoid syndrome treatment with octreotide alone and in combination with alpha-interferon. Acta Oncol. 1993;32(2):225-9. PMID: 7686765. https://doi.org/10.3109/02841869309083916.

13. O'Toole D, Ducreux M, Bommelaer G, et al. Treatment of carcinoid syndrome: A prospective crossover evaluation of lanreotide versus octreotide in terms of efficacy, patient acceptability, and tolerance. Cancer 2000;88(4):770-6. PMID: 10679645. https://doi.org/10.1002/ (sici)1097-0142(20000215)88:4<770::aid-cncr6>3.0.co;2-0.

14. Anthony L, Johnson D, Hande K, et al. Somatostatin analogue phase I trials in neuroendocrine neoplasms. Acta Oncol. 1993;32(2): 217-23. PMID: 7686764. https://doi.org/10.3109/02841869309083915.

Authors are required to accomplish, sign and submit scanned copies of the JAFES Author Form consisting of: (1) Authorship Certification, that authors contributed substantially to the work, that the manuscript has been read and approved by all authors, and that the requirements for authorship have been met by each author; (2) the Author Declaration, that the article represents original material that is not being considered for publication or has not been published or accepted for publication elsewhere, that the article does not infringe or violate any copyrights or intellectual property rights, and that no references have been made to predatory/suspected predatory journals; (3) the Author Contribution Disclosure, which lists the specific contributions of authors; (4) the Author Publishing Agreement which retains author copyright, grants publishing and distribution rights to JAFES, and allows JAFES to apply and enforce an Attribution-Non-Commercial Creative Commons user license; and (5) the Conversion to Visual Abstracts (*optional for original articles only) to improve dissemination to practitioners and lay readers Authors are also required to accomplish, sign, and submit the signed ICMJE form for Disclosure of Potential Conflicts of Interest. For original articles, authors are required to submit a scanned copy of the Ethics Review Approval of their research as well as registration in trial registries as appropriate. For manuscripts reporting data from studies involving animals, authors are required to submit a scanned copy of the Institutional Animal Care and Use Committee approval. For Case Reports or Series, and Images in Endocrinology, consent forms, are required for the publication of information about patients; otherwise, appropriate ethical clearance has been obtained from the institutional review board. Articles and any other material published in the JAFES represent the work of the author(s) and should not be construed to reflect the opinions of the Editors or the Publisher. 